\title{
A case of a new Lynch Syndrome variant with a deletion, c.1024_1026, in the MSH2 gene in a Chinese family
}

\section{Zhe Zhao}

Chinese Academy of Medical Sciences and Peking Union Medical College

Lu Li

Health Service Department of the Guard Bureau of the Joint Staff Department

Lin Dong

Chinese Academy of Medical Sciences and Peking Union Medical College

Jia Jia

Chinese Academy of Medical Sciences and Peking Union Medical College

$\mathrm{Ke} \mathrm{Su}$

Genetron Health (Beijing) Co.Ltd.

Hua Bai

Chinese Academy of Medical Sciences and Peking Union Medical College

Jie Wang ( $\nabla$ zlhuxi@163.com )

Chinese Academy of Medical Sciences and Peking Union Medical College https://orcid.org/0000-00025602-0487

\section{Case report}

Keywords: DNA Mismatch Repair, Hereditary Nonpolyposis Colorectal Neoplasms, Immunotherapy, MutS Homolog 2 Protein, Skin Neoplasms

Posted Date: August 3rd, 2020

DOl: https://doi.org/10.21203/rs.3.rs-50589/v1

License: (c) (i) This work is licensed under a Creative Commons Attribution 4.0 International License.

Read Full License 


\section{Abstract \\ Background}

Multiple genetic variations have been identified in mismatch repair genes for Lynch Syndrome. However, diagnosis of Lynch Syndrome and its subtypes in patients with atypical cancer types still remains challenging. Little is known about Lynch syndrome-related renal carcinoma and related genes. We present a case of renal carcinoma with multiple primary skin tumors, with a new Lynch Syndrome variant with a deletion in a Chinese family.

\section{Case presentation:}

The patient was a 60-year-old Chinese male with a history of Lynch Syndrome-related renal carcinoma with repeatedly multiple primary skin tumors. Immunohistochemistry revealed loss of MSH2 and MSH6 proteins. Sequencing of mismatch repair genes revealed a previously unknown germline $M S H 2$ mutation (c.1024_1026 del) resulting in an amino acid deletion (p. V342del). This variant was co-segregated among the carcinoma-affected family members. After 6 cycles of immunotherapy, a marked regression of the skin tumor was obtained.

\section{Conclusions}

We clarify the pathogenic significance of this new mutation and suggest immunotherapy for patients with this subtype of Lynch Syndrome.

\section{Background}

Lynch Syndrome (LS) is an autosomal dominant disorder, previously known as hereditary nonpolyposis colorectal cancer, that carries a high risk of colorectal and other cancers [1, 2]. The pathogenesis is associated with DNA mismatch repair (MMR) gene mutations including those in $M L H 1, M S H 2, M S H 6$, $P M S 2$, and EPCAM $[3,4]$. These mutations cause deficiencies in DNA repair, resulting in a high frequency of replication errors. Genetic analysis and detection of protein expression by immunohistochemistry (IHC) are important in diagnosing LS $[5,6]$.

Approximately 1 in 350 individuals has LS [7], and different subtypes have varying clinical symptoms and pathological features. Of the individuals with LS, 9.2\% are affected by Muir-Torre syndrome (MTS) [8]. MTS is primarily related to mutations in $\mathrm{MSH} 2$ and is characterized by skin tumors (such as sebaceous adenomas, skin squamous cell carcinomas) [9]. However, MTS-related primary renal carcinoma with skin tumors is rarely reported. We report a case of LS originating in renal cell carcinoma with multiple primary tumors and a previously unknown MSH2 mutation (c.1024_1026 del). 


\section{Case Presentation}

The proband has a history of various cancers. In 2001, at the age of 41 years, he was diagnosed with left kidney clear cell carcinoma; in 2008, with right lung clear cell carcinoma due to renal tumor metastasis; and in 2010 , with bone metastases. In 2019, he had a rectal submucosal neuroendocrine tumor. $\mathrm{He}$ received the standard treatment each time.

In addition to the internal carcinomas, the proband also presented sequentially with multiple neoplasms located on the skin of the head and neck or upper chest, with faster growth starting in 2013 (Fig. 1). The pathology on excised skin lesions revealed primary benign and malignant tumors, including skin squamous cell carcinoma, keratoacanthoma, sebaceous adenoma, and sebaceous gland carcinoma (Additional file 1). Starting in May 2019, the proband had recurring appearance of skin tumors, at a rate of 15-17 per month. The tumors were removed and diagnosed as primary skin malignant tumors by pathological analysis.

\section{Family history}

One brother of the proband died of gastric cancer and three sisters were all diagnosed with endometrial cancer, with one case accompanied by metachronous rectal cancer. Only one brother was cancer-free. In addition, a daughter of one of his sisters was also diagnosed with endometrial cancer at the age of 47. Because of the family history of LS-like cancers and skin lesions, MTS, a subtype of LS, was considered as a diagnosis. Comprehensive genetic testing was performed, and genetic counseling was offered.

\section{Laboratory tests}

MMR protein expression in the proband's visceral and reduplicative skin neoplasms (Additional file 2) was assessed by IHC using MaxVisionTM primary antibodies (anti-MLH1, MAB-0789; anti-PMS2, RMA0775; anti-MSH2, MAB-0836; anti-MSH6, RMA-0770), which revealed the absence of MSH2 and MSH6 expression (Fig. 2). Microsatellite instability (MSI) analysis of genomic DNA with a 5-mononucleotide marker panel (BAT-25, BAT-26, NR-21, NR-24, and MONO-27), and PCR products analyzed by capillary electrophoresis using an ABI 3130xI Genetic Analyzer (Applied Biosystems, Forster City, CA) showed inconsistent results, with MSI-high for the chest squamous cell carcinoma (Additional file 2). Whole exon genetic tests of the proband's multiple tumor tissues and peripheral blood were executed using nextgeneration sequencing (Additional file 2), which showed a germline heterozygous deletion (c.1024_1026 del) in exon 6 of MSH2 on chromosome 2, resulting in a deletion of the p.V342 amino acid. This autosomal dominant mutation excludes promoter methylation and has not been reported previously.

IHC tests of tumor tissues from the proband's siblings and niece also showed the loss of MSH2 and MSH6 proteins, all with microsatellite stable results (Additional file 3). The same $\mathrm{MSH} 2$ mutation (c.1024_1026 del, p.V342del) was detected in samples from the affected siblings and niece, but not those from the cancer-free sibling (Additional file 3), supporting the pathogenic role of this $M S H 2$ variant. 
Further analysis showed that the mutation had been inherited for three consecutive generations in the family (Fig. 3).

\section{Clinical diagnosis}

Consideration of the multi-primary cancers, the family history, and the genetic testing results led to the diagnosis of LS subtype MTS, based on the Amsterdam II criteria. The MSH2 mutation (c.1024_1026del) will be documented in the genetic database.

\section{Clinical treatment}

Based on the result of MMR gene defects and MSI-high, the proband was treated with sintilimab, an inhibitory antibody that binds to PD-1, at $200 \mathrm{mg}$ per 3-week cycle. After 6 cycles, there were no new skin tumors for nearly 5 months, and the size of the largest skin tumor decreased from $0.9 \times 0.6$ to $0.2 \times$ $0.1 \mathrm{~cm}$ (Fig. 1). The patient's condition is stable, and the efficacy of immunotherapy is being monitored.

\section{Discussion And Conclusions}

The presence of multiple sebaceous glands is a sign of MMR gene defects and may be a useful clinical parameter for diagnosing the MTS subtype of LS [10]. Approximately $70-75 \%$ of LS cases are caused by mutations in $M S H 2$ and $M L H 1$ [11]. The in-frame deletion of $M S H 2$ identified in this study (c.1024_1026 del) has not been reported previously. We have proposed the correlation of the gene with LS/MTS, which clarifies the prognosis of the proband, and provides guidance for early cancer screening for the family descendants. Future development of in silico analysis may facilitate opportune screening, diagnosis, and prevention.

Irradiation and excision of skin tumors and the use of isotretinoin to prevent recurrence with monitoring for early detection of visceral tumors may be beneficial as treatments for LS/MTS $[12,13]$. Immunotherapy in LS is mostly used for the treatment of multiple visceral metastases [14], and there is lack of evidence for the treatment of repeated skin tumors. Our results suggest that LS/MTS patients with difficult-to-control skin tumors can benefit from immunotherapy, and the treatment possibly has a preventive effect on the progress of subsequent visceral cancers, which raises a challenge to monitor the effectiveness of immunotherapy. Further studies are needed to provide more conclusive evidence.

In conclusion, the in-frame deletion of MSH2 (c.1024_1026 del) is supposed to be the pathogenic new mutation for LS/MTS, and immunotherapy is a treatment strategy that can be considered for patients with this subtype of Lynch Syndrome.

\section{Abbreviations}

Lynch Syndrome (LS), Muir-Torre syndrome (MTS), microsatellite instability (MSI), mismatch repair (MMR), immunohistochemistry (IHC), tumor mutational burden (TMB). 


\section{Declarations}

\section{Ethics approval and consent to participate}

All procedures were performed in accordance with and followed the ethical standards of the Human Laboratory Responsibility Committee of the Cancer Hospital of the Chinese Academy of Medical Sciences (Beijing, China) and complied with the 2013 revised Declaration of Helsinki. Informed consent was obtained from all patients enrolled in the study.

\section{Consent for publication}

The proband and his family have approved publication of this report.

\section{Availability of data and materials}

The datasets used and/or analyzed during the current study are available from the corresponding author on reasonable request.

\section{Competing interests}

The authors declare that they have no competing interests.

\section{Funding}

This work was supported by National key research and development project 2019YFC1315700; the National Natural Sciences Foundation Key Program [81630071]; CAMS Innovation Fund for Medical Sciences (CIFMS 2016-I2M-3-008); Aiyou foundation (KY201701); Ministry of Education Innovation Team development project (IRT-17R10); and CAMS Key lab of translational research on lung cancer (2018PT31035).

\section{Authors' contributions}

$\mathrm{ZZ}$ and HB were responsible of the clinical management of the patient and acquisition of data, and helped to draft the manuscript. ZZ was a major contributor in writing the manuscript. LL was responsible of samples acquisition and interpreted the sequencing data on tissues and blood from the family. LD and $\mathrm{JJ}$ were responsible of the pathological diagnosis and immunohistochemical analysis. KS was responsible for the genetic testing of tumor tissues or peripheral blood. JW was responsible of the clinical management, interpretation of the comprehensive data, and scientific revision. All authors read and approved the final manuscript. 


\section{Acknowledgements}

We thank the patient and his family. Genetron Health (Beijing) Co. Ltd provided the sequencing service for this research.

\section{References}

1. Cohen SA, Pritchard CC, Jarvik GP. Lynch Syndrome: From Screening to Diagnosis to Treatment in the Era of Modern Molecular Oncology. Annu Rev Genomics Hum Genet. 2019;20:293-307. doi:10.1146/annurev-genom-083118-015406.

2. Lynch HT, Snyder CL, Shaw TG, Heinen CD, Hitchins MP. Milestones of Lynch syndrome: 1895-2015. Nat Rev Cancer. 2015;15(3):181-94. doi:10.1038/nrc3878.

3. Gray PN, Tsai P, Chen D, Wu S, Hoo J, Mu W, et al. TumorNext-Lynch-MMR: a comprehensive next generation sequencing assay for the detection of germline and somatic mutations in genes associated with mismatch repair deficiency and Lynch syndrome. Oncotarget. 2018;9(29):20304-22. doi:10.18632/oncotarget.24854.

4. Duraturo F, Liccardo R, De Rosa M, Izzo P. Genetics, diagnosis and treatment of Lynch syndrome: Old lessons and current challenges. Oncol Lett. 2019;17(3):3048-54. doi:10.3892/ol.2019.9945.

5. Tognetto A, Pastorino R, Castorina S, Condorelli DF, DeCensi A, De Vito C, et al. The Current Practice of Lynch Syndrome Diagnosis and Management in Italy: A Qualitative Assessment. Public Health Genomics. 2019;22(5-6):189-207. doi:10.1159/000504305.

6. Ismael NE, El Sheikh SA, Talaat SM, Salem EM. Mismatch Repair Proteins and Microsatellite Instability in Colorectal Carcinoma (MLH1, MSH2, MSH6 and PMS2): Histopathological and Immunohistochemical Study. Open Access Maced J Med Sci. 2017;5(1):9-13. doi:10.3889/oamjms.2017.003.

7. $10.1053 /$ j.gastro.2010.04.024

Boland CR, Shike M. Report from the Jerusalem workshop on Lynch syndrome-hereditary nonpolyposis colorectal cancer. Gastroenterology. 2010;138(7):2197.e1-7. doi:

10.1053/j.gastro.2010.04.024.

8. South CD, Hampel H, Comeras I, Westman JA, Frankel WL, de la Chapelle A. The frequency of MuirTorre syndrome among Lynch syndrome families. J Natl Cancer Inst. 2008;100(4):277-81. doi:10.1093/jnci/djm291.

9. Le S, Ansari U, Mumtaz A, Malik K, Patel P, Doyle A, et al. Lynch Syndrome and Muir-Torre Syndrome: An update and review on the genetics, epidemiology, and management of two related disorders. Dermatol Online J. 2017;23(11):13030/qt8sg5w98j.

10. John AM, Schwartz RA. Muir-Torre syndrome (MTS): An update and approach to diagnosis and management. J Am Acad Dermatol. 2016;74(3):558-66. doi:10.1016/j.jaad.2015.09.074. 
11. Bouvet D, Bodo S, Munier A, Guillerm E, Bertrand R, Colas C, et al. Methylation Tolerance-Based Functional Assay to Assess Variants of Unknown Significance in the MLH1 and MSH2 Genes and Identify Patients With Lynch Syndrome. Gastroenterology. 2019;157(2):421-31. doi:10.1053/j.gastro.2019.03.071.

12. Hood IC, Qizilbash AH, Salama SS, Young JE, Archibald SD. Sebaceous carcinoma of the face following irradiation. Am J Dermatopathol. 1986;8(6):505-8. doi:10.1097/00000372-19861200000009.

13. Graefe T, Wollina U, Schulz H, Burgdorf W. Muir-Torre syndrome - treatment with isotretinoin and interferon alpha-2a can prevent tumour development. Dermatology. 2000;200(4):331-3. doi:10.1159/000018399.

14. Feng Y, Cao Y, Yuan M, Chen R, Ji X, Hu X. Different responses to anti-programmed cell death protein 1 (PD-1) immunotherapy in a patient with Lynch syndrome and metachronous urothelial and colon cancer: A case report. Oncol Lett. 2019;18(5):5085-90. doi:10.3892/ol.2019.10909.

\section{Figures}



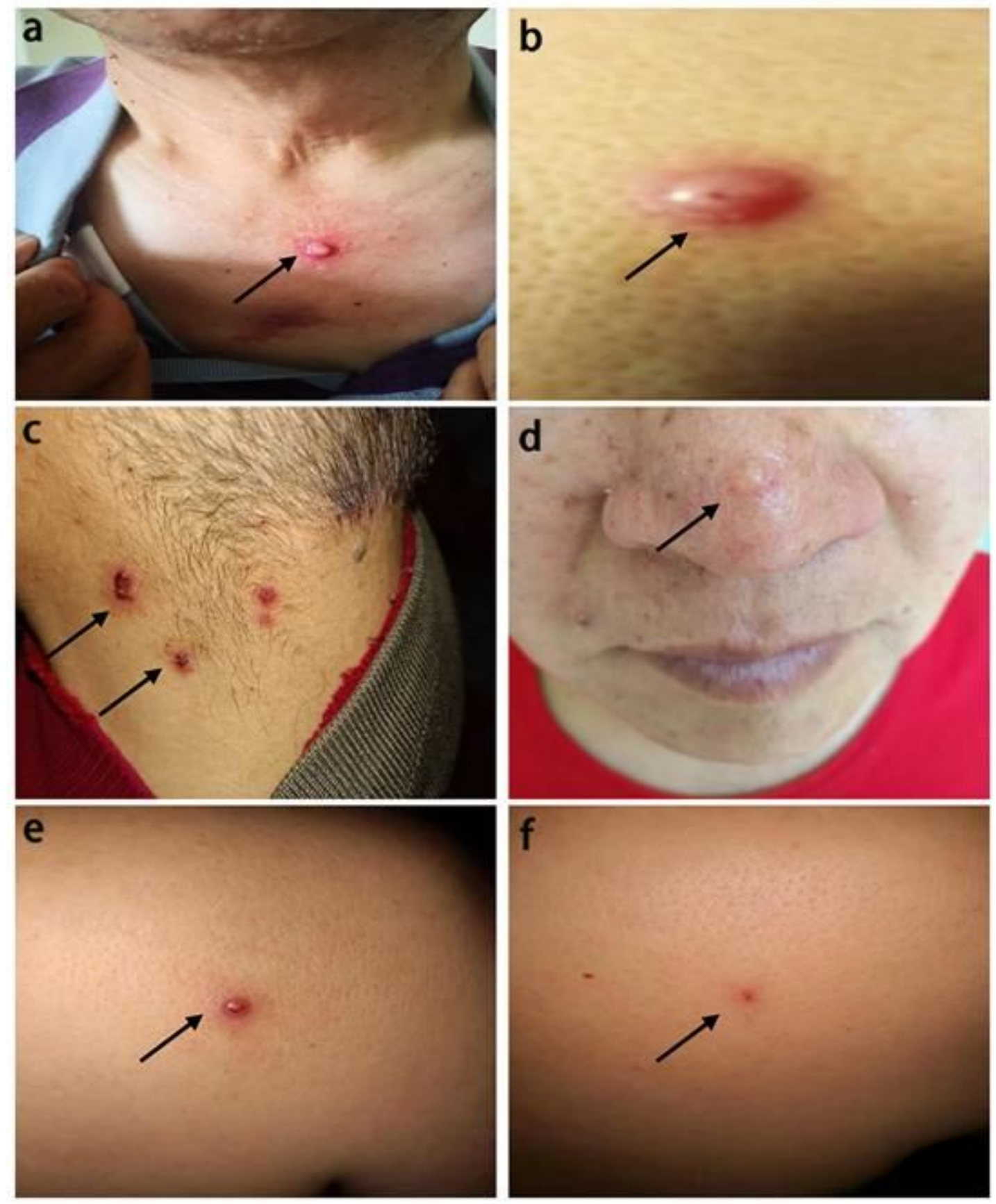

\section{Figure 1}

Clinical manifestations of the proband's skin tumors Panels a- $d$ show examples of the proband's skin tumors. The patient presented with repeated skin tumors over the years on the chest (a), back (b), neck (c), and face (d), with pathological analysis after surgical resection showing squamous cell carcinoma (a), hyperplastic squamous cell carcinoma (b), and sebaceous adenomas (c and d). Panels e and $\mathrm{f}$ are examples of skin tumor changes before and after immunotherapy. The nodule in the back shoulder was one of multiple skin tumors before immunotherapy $(0.9 \times 0.6 \mathrm{~cm})(\mathrm{e})$, which shrunk significantly $(0.2 \times$ $0.1 \mathrm{~cm})(\mathrm{f})$. 

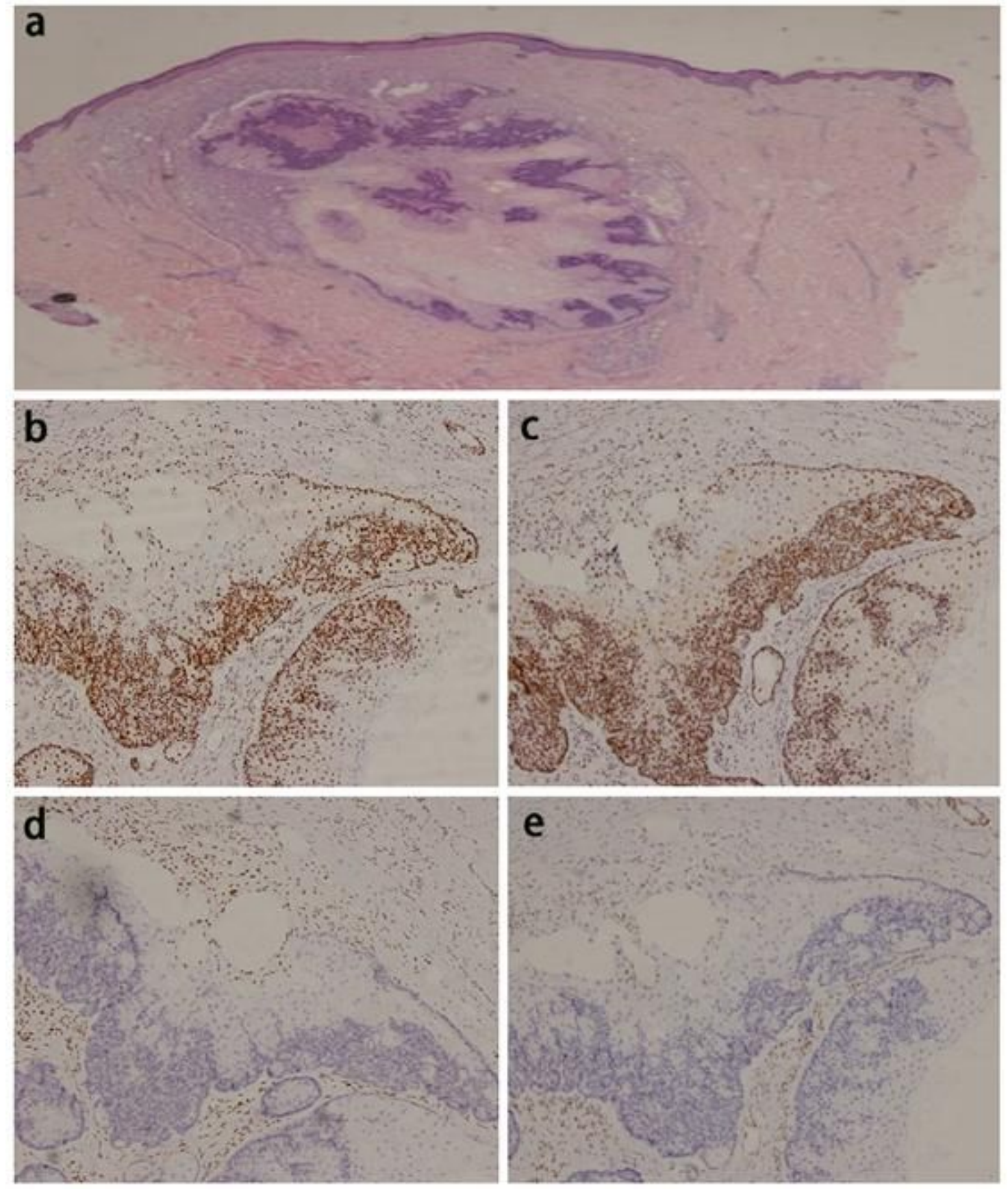

\section{Figure 2}

Immunohistochemistry of skin tumors IHC of a skin tumor (pathology for the sebaceous gland adenoma) revealed the loss of MSH2 and MSH6 proteins. (a) HE-stained section, 20x; (b) MLH1, 100x; (c) PMS2, 100x; (d) MSH2, 100x; (e) MSH6, 100x. 
II

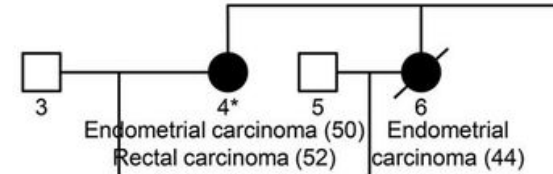

III
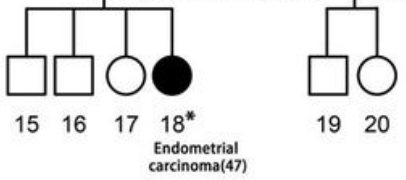

1920

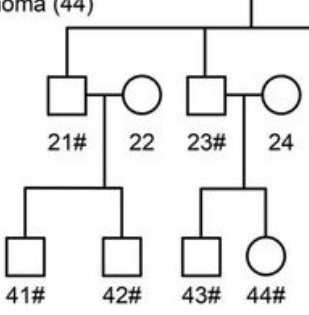

IV

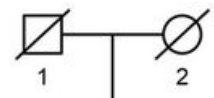

$$
2
$$

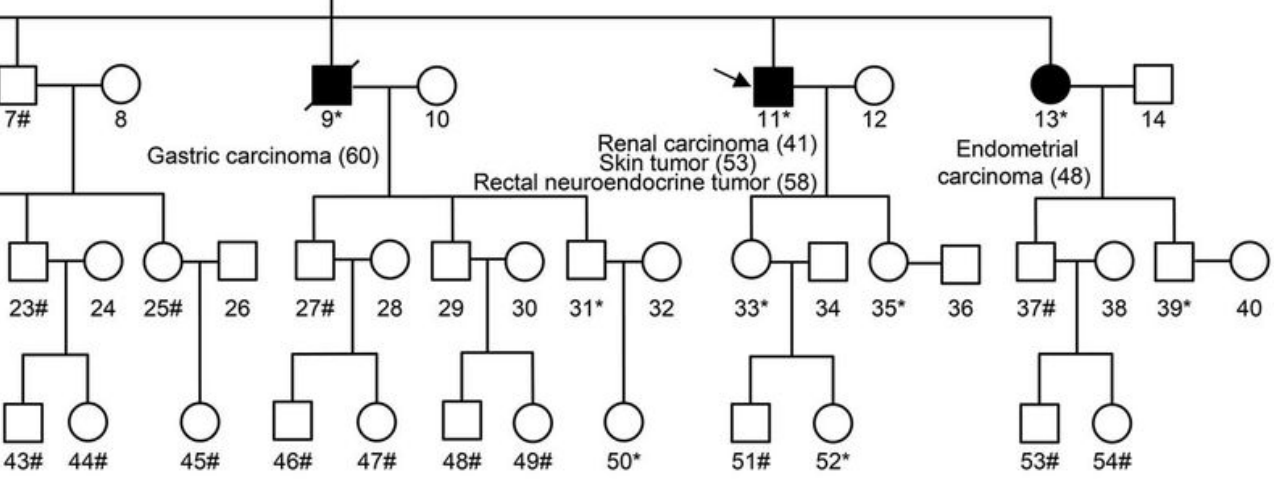

Figure 3

Pedigree of family with MSH2 c.1024_1026 del The arrow $(\rightarrow)$ indicates the proband. Squares and circles denote males and females, respectively. Roman numerals indicate generations. Black solids represent tumor patients. The numbers in the brackets represent the age of onset of the disease. The asterisk (*) indicates a carrier of the MSH2 gene c.1024_1026 del mutation. The pound (\#) indicates a non-carrier. The remaining family members have not been tested for $\mathrm{MSH} 2$ mutations.

\section{Supplementary Files}

This is a list of supplementary files associated with this preprint. Click to download.

- SupportingFileAdditionalfile3.docx

- SupportingFileAdditionalfile3.docx

- SupportingFileAdditionalfile2.docx

- SupportingFileAdditionalfile2.docx

- SupportingFileAdditionalfile1.docx

- SupportingFileAdditionalfile1.docx 